T. OGATA

KODAI MATH. J.

5 (1982), 266-282.

\title{
OPERATIONAL CALCULUS OF TWO VARIABLES
}

\author{
By TAKASHI OgatA
}

\section{Introduction.}

J. Mikusiński [2] has introduced a simple and complete operational calculus to obtain the solutions of linear ordinary differential equations with constant coefficients. The significance of operational calculus is that operators are regarded as convolution quotient, that is, functions and differential operator are elements of the same set. Moreover, he has discussed the linear partial differential equations of two variables with constant coefficients. In [2], the solutions were obtained by the traditional method of testing solutions by substituting the exponential functions, not by the systematic one as operational calculus. The difficulty of his method is that no prospective insight for obtaining the particular solutions has been given. In this paper, we discuss the linear partial differential equations of two variables with constant coefficients by the same systematic method as Mikusiński's operational calculus by imposing some restrictions on the constant part on the right hand side (cf. (2.7)).

We believe that the results obtained by such a way give a new development to the theory of Mikusiński's operational calculus.

In the last section, we show some well-known examples.

\section{Operational calculus.}

Let $\hat{k}$ be an algebrically closed field of characteristic 0 and $A=\hat{k}[[\lambda]]$ be the module of the formal power series of a variable $\lambda$ with coefficients in $\hat{k}$. Henceforth, we denote each element of $\mathcal{A}$ by $P(\lambda)$, or simply $P=\left\{\sum_{\nu=0}^{\infty} p_{\nu} \lambda^{\nu}\right\}$ (instead of usual notation $\left.\sum_{\nu=0}^{\infty} p_{\nu} \lambda^{\nu}\right)$ where $p_{\nu} \in \hat{k}(\nu=0,1,2, \cdots)$.

Definition 2.1. Multiplication in $A$ is defined by

$$
(P Q)(\lambda)=\left\{\sum_{\rho=0}^{\infty}\left(\sum_{\rho=\nu+\mu} \frac{\nu ! \mu !}{(\rho+1) !} p_{\nu} q_{\mu}\right) \lambda^{\rho+1}\right\}
$$

where $P=\left\{\sum_{\nu=0}^{\infty} p_{\nu} \lambda^{\nu}\right\}$ and $Q=\left\{\sum_{\mu=0}^{\infty} q_{\mu} \lambda^{\mu}\right\}$.

Recerved January 13, 1981 
Proposition 2.2. By usual addition and above multiplication $A$ forms an integral domain without unit element.

Proof. We show that $A$ has no zero devisor. If $P \neq 0$ and $Q \neq 0$, there exist $\nu_{0}=\min \left\{\nu ; p_{\nu} \neq 0\right\}$ and $\mu_{0}=\min \left\{\mu ; q_{\mu} \neq 0\right\}$. Then coefficient of $\lambda^{\nu_{0}+\mu_{0}+1}$ in $P Q$ is $\left(\left(\nu_{0} ! \mu_{0} !\right) /\left(\nu_{0}+\mu_{0}+1\right) !\right) p_{\nu_{0}} q_{\mu_{0}}$ which is nonzero. The other parts are shown by the same way as Mikusiński [2].

By Proposition 2.2, we can construct the quotient field $Q(\mathcal{A})$ of the ring $A$. An element of $Q(\mathcal{A})$ is called operator. For the special operators in $Q(\mathcal{A})$, we have integral operator $L=\{1\}, \hat{k}$-operator (each element of $\hat{k}$ ) which corresponds to numerical operator and differentıal operator $S=1 / L$ as the same as Mikusiński's cases.

Definition 2.3. The mapping $\partial_{\lambda}$ of $\mathcal{A}$ into itself is defined by

$$
\partial_{\lambda}\left\{p_{0}+p_{1} \lambda+p_{2} \lambda^{2}+p_{3} \lambda^{3}+\cdots\right\}=\left\{p_{1}+2 p_{2} \lambda+3 p_{3} \lambda^{2}+\cdots\right\} .
$$

Moreover, for any positive integer $n, \partial_{\lambda}^{n} P$ is defined inductively by $\partial_{\lambda}^{n} P=$ $\partial_{\lambda}\left(\partial_{\lambda}^{n-1} P\right)$.

In this section, we denote $\partial_{\lambda}$ by $\partial$ for brevity. By Definition 2.3 we have, for any $P=\left\{\sum_{\nu=0}^{\infty} p_{\nu} \lambda^{2}\right\}$,

$$
L(\partial P)=P-L \cdot P(0) .
$$

where $P(0)=p_{0}$ is $\hat{k}$-operator. Hence, we have

$$
S P=\partial P+P(0) \text {. }
$$

By the induction, we can get the following general formula.

Proposition 2.4. For $n=1,2,3, \cdots$,

$$
S^{n} P=\partial^{n} P+\left(\partial^{n-1} P\right)(0)+S \cdot\left(\partial^{n-2} P\right)(0)+\cdots+S^{n-1} \cdot P(0)
$$

For any $p \in \hat{k}$, we define the element $\left\{e^{p \lambda}\right\}$ of $A$ by

$$
\left\{e^{p \lambda}\right\}=\left\{1+p \lambda+\frac{(p \lambda)^{2}}{2 !}+\frac{(p \lambda)^{3}}{3 !}+\cdots\right\} .
$$

Then, by (2.2), we have $S\left\{e^{p \lambda}\right\}=p\left\{e^{p \lambda}\right\}+1$. Hence,

$$
\frac{1}{(S-p)}=\left\{e^{p \lambda}\right\}
$$

Generally, we have

LEMMA 2.5 .

$$
\frac{1}{(S-p)^{n}}=\left\{\frac{\lambda^{n-1}}{(n-1)}, e^{p \lambda}\right\} \quad \text { for } n=1,2,3, \cdots .
$$


Proof. We show by the induction.

$$
\partial\left\{\frac{\lambda^{n}}{n !} e^{p \lambda}\right\}=\partial\left\{\sum_{\nu=0}^{\infty} \frac{p^{\nu} \lambda^{\nu+n}}{n ! \nu !}\right\}=\left\{\frac{\lambda^{n-1}}{(n-1) !} e^{p \lambda}\right\}+\left\{\frac{p \lambda^{n}}{n !} \cdot e^{p \lambda}\right\} .
$$

Hence, $S\left\{\frac{\lambda^{n}}{n !} e^{p \lambda}\right\}=\frac{1}{(S-p)^{n}}+p\left\{\frac{\lambda^{n}}{n !} e^{p \lambda}\right\}$. This shows that

$$
\frac{1}{(S-p)^{n+1}}=\left\{\frac{\lambda^{n}}{n !} e^{p \lambda}\right\}
$$

Let's consider the following fractional expression in $Q(\mathcal{A})$.

$$
R(S)=\frac{q_{m} S^{m}+q_{m-1} S^{m-1}+\cdots+q_{0}}{p_{n} S^{n}+p_{n-1} S^{n-1}+\cdots+p_{0}},
$$

where $p_{\imath}, q_{j} \in \hat{k}(i=1, \cdots, n ; j=1, \cdots, m)$ and $p_{n} \neq 0$, which is called a ratıonal operator. In the same way as Mikusiński [2], we get the following.

Proposition 2.6. Suppose $m<n$, then $R(S) \in \mathcal{A}$.

Now, the operational calculus provides us with convenient method of solving the linear differential equations with constant coefficients. Consider the following formal linear differential equation of the $n$-th degree.

$$
p_{n} \partial^{n} X+p_{n-1} \partial^{n-1} X+\cdots+p_{1} \partial X+p_{0} X=P,
$$

where $p_{i} \in \hat{k}(i=0,1, \cdots, n), p_{n} \neq 0$ and $P \in \mathcal{A}$.

We seek a solution $X$ in $A$ satisfying the following initial conditions

$$
X(0)=r_{0}, \quad(\partial X)(0)=r_{1}, \cdots,\left(\partial^{n-1} X\right)(0)=r_{n-1},\left(r_{i} \in \hat{k}\right) .
$$
form,

By virtue of the general formula (2.3), equation (2.7) may be written in the

$$
\left(p_{n} S^{n}+p_{n-1} S^{n-1}+\cdots+p_{0}\right) X=q_{n-1} S^{n-1}+\cdots+q_{0}+P,
$$

where

$$
q_{\imath}=p_{\imath+1} r_{0}+p_{\imath+2} r_{1}+\cdots+p_{n} r_{n-\imath-1} \quad(i=0,1, \cdots, n-1) .
$$

Hence, we immediately find that

$$
X=\frac{q_{n-1} S^{n-1}+\cdots+q_{0}}{p_{n} S^{n}+\cdots+p_{0}}+\frac{P}{p_{n} S^{n}+\cdots+p_{0}} .
$$

Applying decomposition into simple fractions, we obtain the solution in $\mathcal{A}$ by Proposition 2.6. 


\section{The field of Puiseux series.}

Let $k$ be a field of characteristic 0 . In this section, we consider the following power series of fractional power of a variable $u$ with coefficients in $k$.

$$
p=\alpha_{1} u^{r_{1}}+\alpha_{2} u^{r_{2}}+\alpha_{3} u^{r_{3}}+\cdots, \quad \text { where } \alpha_{i} \in k \text { and } \alpha_{i} \neq 0 .
$$

A power series of the form (3.1) satisfying the following conditions (C1) and (C2) is called a Puiseux serzes.

(C1) Each $r_{\imath}$ are rational numbers such that $r_{i}<r_{\imath+1}$.

(C2) For each $r_{\imath}$, we denote $r_{\imath}=n_{\imath} / m_{\imath}$ such that integers $n_{\imath}, m_{i}(>0)$ are relatively prime. Then there exists positive integer $m$ such that $m_{i}<m$ for all $\imath$.

Let $e=e(p)$ be the least common multiple $\left[m_{1}, m_{2}, m_{3}, \cdots\right]$ of $m_{1}, m_{2}, m_{3}, \cdots$. From (C2), $e$ is finite. Hence, (3.1) is expressed as the following form.

$$
\alpha_{1}\left(u^{1 / e}\right)^{e r_{1}}+\alpha_{2}\left(u^{1 / e}\right)^{e r_{2}}+\cdots,
$$

where each $e r_{\imath}$ are integers. In the case of need, we denote Puiseux series by the following form,

$$
\sum_{n \geqq-n_{0}} \beta_{n}\left(u^{1 / e}\right)^{n}
$$

where $e>0, n_{0}>0$ and $n$ are integers.

Let $k\{u\}$ be the set of all Puiseux series. Then $k\{u\}$ forms a field with respect to usual addition and multiplication. It is known that $k\{u\}$ is algebrically closed if $k$ is an algebrically closed field of characteristic 0 (cf. [1]). Hence, $C\{l\}$ is algebrically closed where $\boldsymbol{C}$ is the complex number field and $l$ a variable. Put $s=l^{-1}$ and let $\boldsymbol{C}(l)$ or $\boldsymbol{C}(s)$ be the rational function field of variable $l$ or $s$, respectively. We have obviously $\boldsymbol{C}(s)=\boldsymbol{C}(l) \subset \boldsymbol{C}\{l\}$.

Definition 3.1. For any $p(\neq 0) \in \boldsymbol{C}\{l\}$ such that $p=\sum_{\imath=1}^{\infty} \alpha_{\imath} l^{r_{i}}\left(\alpha_{\imath} \neq 0\right)$, we define $\nu(p)=r_{1}$ and $\nu(0)=\infty$.

Then $\nu(p)$ is a valuation on $C\{l\}$.

For $p_{i} \in \boldsymbol{C}\{l\} \quad(i=1,2, \cdots)$, suppose that

$$
\begin{aligned}
& \left\{e\left(p_{\imath}\right) ; \imath=1,2, \cdots\right\} \text { is bounded, } \\
& \nu\left(p_{\imath}\right)<\nu\left(p_{\imath+1}\right) \quad \text { for each } \imath,
\end{aligned}
$$

then we define $\sum_{\imath=1}^{\infty} p_{\imath}$ as the following. We denote $p_{\imath}=\sum_{j=1}^{\infty} \alpha_{\imath \jmath} l^{r_{\imath \jmath}}\left(\alpha_{\imath 1} \neq 0\right)$ for each $i$. From (3.5), $r_{11}<r_{\imath}$ for any $i$ and $j$. Arranging $\left\{r_{\imath j}\right\}(i, j=1,2, \cdots)$, we have the sequence $\left\{r_{k}\right\}$ such that $r_{11}=r_{1}<r_{2}<\cdots$. Because $\left\{r_{\imath j}\right\} \quad(\jmath=1,2, \cdots)$ is a 
subsequence of $\left\{r_{k}\right\}$, each $p_{\imath}$ is written by $\sum_{j=1}^{\infty} \beta_{i j} r^{r}$, where

$$
\begin{gathered}
\beta_{i \jmath}=\alpha_{\imath k} \quad \text { when } r_{\jmath}=r_{\imath k} \text { for some } k, \\
\beta_{\imath \jmath}=0 \quad \text { when } r_{j} \neq r_{\imath k} \text { for all } k .
\end{gathered}
$$

For each $r_{\imath}$, denoting $r_{\imath}=n_{\imath} / m_{\imath}$ such that integers $n_{\imath}, m_{i}(>0)$ are relatively prime and $e=\left[m_{1}, m_{2}, \cdots\right]$, we have $e \leqq\left[e\left(p_{1}\right), e\left(p_{2}\right), \cdots\right]$. So, from (3.4), $e$ is finite. Because each $e r_{\imath}$ are integers and $e r_{11}<e r_{\imath \jmath}$, the number of negative ones in $\left\{r_{i}\right\}$ is finite. On the other hand, for any $r$ of $\left\{r_{k}\right\}$, we set $I_{r}=\left\{i \in N ; r=r_{\imath}\right.$ for some $j\}$. Then there exists $k$ such that $e \nu\left(p_{k-1}\right)<e r \leqq e \nu\left(p_{k}\right)$ from (3.5). Hence, the number of $I_{r}$ is less than $k$ and $\alpha_{\jmath}=\sum_{i=1}^{\infty} \beta_{i \jmath}=\sum_{i \in I_{r_{j}}} \beta_{i j}$ is finite sum. So, we define $\sum_{i=1}^{\infty} p_{\imath}=\sum_{j=1}^{\infty} \alpha_{\jmath} l^{r}$. From the above argument, we have

LEMMA 3.2. For $p_{\imath}(i=1,2, \cdots)$ which satısfy the conditzons (3.4), (3.5), as above, we have $\sum_{i=1}^{\infty} p_{i} \in C\{l\}$.

Applying Lemma 3.2, we show two examples.

ExAmple 1. For $p \in \boldsymbol{C}\{l\}$ such that $\nu(p)>0$, we have

$$
\nu\left(p^{i}\right)=\imath \nu(p) \quad(i=0,1,2, \cdots),
$$

which shows that $\left\{p^{i}\right\}$ satisfies the condition (3.5) and we get easily that $e\left(p^{i}\right)$ $\leqq e(p)$ for any $\imath$. Let $\left(\begin{array}{l}\lambda \\ k\end{array}\right)$ be the formal binomial coefficient, that is, for some real number $\lambda$ and some positive integer $k$,

$$
\left(\begin{array}{l}
\lambda \\
k
\end{array}\right)=\frac{\lambda(\lambda-1)(\lambda-2) \cdots(\lambda-k+1)}{k !} \text { and }\left(\begin{array}{l}
\lambda \\
0
\end{array}\right)=1 \text {. }
$$

By Lemma 3.2, $\sum_{k=0}^{\infty}\left(\begin{array}{l}\lambda \\ k\end{array}\right) p^{k} \in C\{l\}$. So, we define

$$
(1+p)^{\lambda}=\sum_{k=0}^{\infty}\left(\begin{array}{l}
\lambda \\
k
\end{array}\right) p^{k}
$$

By the properties of binomial coefficient, we get, for any real numbers $\lambda, \mu$ and an integer $n$,

$$
\begin{gathered}
(1+p)^{\lambda}(1+p)^{\mu}=(1+p)^{\lambda+\mu}, \\
\left((1+p)^{\lambda / n}\right)^{n}=(1+p)^{\lambda} .
\end{gathered}
$$

ExAmple 2. Let $p, q \in \boldsymbol{C}\{l\}$ such that $\nu(q)>0$ and $p=\sum_{i=1}^{\infty} \alpha_{i} l^{r_{i}}, q=\sum_{i=1}^{\infty} \beta_{i} l^{u_{\imath}}$ 
$\left(\beta_{1} \neq 0\right)$. Here, we put $q_{1}=\sum_{j=2}^{\infty}\left(\beta_{j} / \beta_{1}\right) l^{u_{j} u_{1}}$, then $\nu\left(q_{1}\right)>0$ and $q=\beta_{1} l^{u_{1}}\left(1+q_{1}\right)$. From example $1,\left(1+q_{1}\right)^{r_{i}}$ is defined and we get

$$
\alpha_{i} q^{r_{i}}=\alpha_{\imath} \beta_{1}^{r_{i}} l^{u_{1} r_{i}}\left(1+q_{1}\right)^{r_{i}},
$$

which is an element of $\boldsymbol{C}\{l\}$. Because of $e\left(\alpha_{i} q^{r_{i}}\right) \leqq e(p) e(q)$ and $\nu\left(\alpha_{i} q^{r_{i}}\right)=r_{i} \nu(q)$ if $\alpha_{i} \neq 0, \sum_{i=1}^{\infty} \alpha_{i} q^{r_{i}} \in \boldsymbol{C}\{l\}$ follows from Lemma 3.2. Hence, we define $p(q) \in \boldsymbol{C}\{l\}$ by

$$
p(q)=\sum_{i=1}^{\infty} \alpha_{i} q^{r_{\imath}}
$$

Definition 3.3. The mapping $\partial_{t}$ of $C\{l\}$ into itself is defined by $\partial_{t}(p)=$ $s p-p(0)$ for any $p=\sum_{i=1}^{\infty} \alpha_{i} l^{r_{i}} \in C\{l\}$, where $s=l^{-1}$, and $p(0)=\alpha_{\imath_{0}}$ or 0 if there exists $r_{\iota_{0}}$ such that $r_{\imath_{0}}=1$ or not, respectively. Moreover, $\partial_{t}^{n}(p)$ is defined inductively by $\partial_{t}^{n}(p)=\partial_{t}\left(\partial_{t}^{n-1}(p)\right)$ for $n=2,3, \cdots$.

From Definition 3.3, we have the following formula by the induction.

Proposition 3.4. For $n=1,2,3, \cdots$,

$$
\partial_{t}^{n}(p)=s^{n} p-s^{n-1} \cdot p(0)-s^{n-2} \cdot\left(\partial_{t} p\right)(0)-\cdots-\left(\partial_{t}^{n-1} p\right)(0) .
$$

\section{The "function elements" of $C\{l\}$.}

Let $a(t)$ be a complex-valued function such that $a(t)=0$ for $t<0$ and $\mathcal{K}$ the set of all such functions which satisfy the following conditions.

(4.1) $a(t)$ has at most a finite number of points of discontinuity in every interval.

(4.2) The integral $\int_{0}^{t}|a(\tau)| d \tau$ has a finite value for every $t>0$.

The equality of two elements $a(t), b(t)$ of $\mathcal{K}$ is defined by the following

$$
a(t)=b(t) \text { if and only if } \int_{0}^{t} a(\tau) d \tau=\int_{0}^{t} b(\tau) d \tau \text { for every } t>0
$$

which is equivalent to that $a(t)$ and $b(t)$ have the same values at every point where both are continuous.

It is known that the convolution of elements of $\mathcal{K}$ is also element of $\mathcal{K}$ (cf. [2], p. 346). Hence, $\mathcal{K}$ forms a ring with usual addition and multiplication by the convolution.

Now, we shall regard some elements of $\boldsymbol{C}\{l\}$ as ones of $\mathcal{K}$. For any $p=\sum_{n \leq-n_{0}} \alpha_{n}\left(l^{1 / e}\right)^{n} \in \boldsymbol{C}\{l\}$, we define a formal power series $p_{z}$ with complex variable $z$ by 


$$
p_{z}=\sum_{n \geq-n_{0}} \alpha_{n} z^{n}
$$

The radius of convergence of $p_{z}$ is denoted by $r\left(p_{z}\right)$. For any natural number $m, p_{z}^{(m)}$ is defined by

$$
p_{z}^{(m)}=\sum_{n \geq-n_{0}} \alpha_{n} z^{m n} .
$$

Let $\mathscr{I}$ be the subset of $\boldsymbol{C}\{l\}$ given by the following

Then we have

$$
\mathscr{I}=\left\{p \in C\{l\} ;(1) \nu(p)>0 \text { and }(2) r\left(p_{z}\right)>0\right\}
$$

Proposition 4.1. I is a subring of $\boldsymbol{C}\{l\}$.

Proof. Let $f, g \in \mathscr{F}$ and $\alpha \in C$. According to the properties of valuation, we have $\nu(\alpha f)>0, \nu(f+g)>0$ and $\nu(f g)>0$. On the other hand, for suitable natural numbers $m_{1}, m_{2}, m_{3}, n_{1}, n_{2}$ and $n_{3}$, we have

$$
(f+g)_{z}^{\left(m_{1}\right)}=f_{z}^{\left(m_{2}\right)}+g_{z}^{\left(m_{3}\right)} \text { and }(f g)_{z}^{\left(n_{1}\right)}=f_{z}^{\left(n_{2}\right)} g_{z}^{\left(n_{3}\right)},
$$

which shows that $r\left((f+g)_{z}\right)>0$ and $r\left((f g)_{z}\right)>0$.

Moreover, $\mathscr{F}$ has the following property.

Proposition 4.2. For any $f, g \in \mathscr{F}, f(g) \in \mathscr{F}$.

Proof. According to example 2 of section 3, $\nu(f(g))=\nu(f) \nu(g)>0$ and $(f(g))_{z}$ $=f_{z}\left(g_{z}\right)$. Hence, we have $r\left((f(g))_{z}\right)>0$.

Let $\Gamma(\lambda)$ be the Euler's gamma function, that is, for a positive real number $\lambda$,

$$
\Gamma(\lambda)=\int_{0}^{\infty} t^{\lambda-1} e^{-t} d t
$$

Proposition 4.3. Suppose that a series $\sum_{n=1}^{\infty} \alpha_{n} z^{n}\left(\alpha_{n} \in C\right)$ has a positive convergent radius $r$, then, for every $\beta>0$, the power series $\sum_{n=1}^{\infty} \alpha_{n} t^{n \beta-1} / \Gamma(n \beta)$ is uniformly convergent in any closed interval $\left[t_{1}, t_{2}\right]$ where $0<t_{1}<t_{2}$. So, this is an element of $\kappa$.

Proof. We consider a natural number $n$ such that $n \beta>2$, so, for any positive number $\alpha>0$,

$$
\begin{aligned}
\Gamma(n \beta) & =\int_{0}^{\infty} t^{n \beta-1} e^{-t} d t>\int_{\alpha}^{\alpha+1} t^{n \beta-1} e^{-t} d t>\int_{\alpha}^{\alpha+1} \alpha^{n \beta-1} e^{-\alpha-1} d t \\
& =\alpha^{n \beta-1} e^{-\alpha-1} .
\end{aligned}
$$

For any $t_{2}>0$, take $\alpha$ such that $t_{2}=\alpha(r / 2)^{1 / \beta}$, then for any $t$ such that $0<t_{1}$ $\leqq t \leqq t_{2}$, we have 
Hence, we have

$$
t_{1}^{n \beta-1}<\alpha^{n \beta-1}(r / 2)^{(n \beta-1) / \beta} .
$$

$$
\left|t^{n \beta-1} / \Gamma(n \beta)\right|<e^{\alpha+1}(r / 2)^{n}(r / 2)^{-1 / \beta} .
$$

From which, the Proposition is proved.

Now, we shall define a mapping $i$ from $\Phi$ to $\mathcal{K}$ as the following. For a positive real number $\lambda$, $\left\{t^{\lambda-1} / \Gamma(\lambda)\right\} \in \mathcal{K}$, so we define, at first,

$$
i\left(l^{\lambda}\right)=\left\{t^{\lambda-1} / \Gamma(\lambda)\right\} \text {. }
$$

By the property of the convolution, we have

$$
i\left(l^{\lambda}\right) i\left(l^{\mu}\right)=i\left(l^{\lambda+\mu}\right),
$$

for any positive real numbers $\lambda, \mu$. Especially, we have $\left(i\left(l^{\lambda}\right)\right)^{n}=i\left(l^{n} \lambda\right)$ for any natural number $n$.

From (4.7), for $f \in \mathscr{F}$ such that $f=\sum_{k=1}^{\infty} \alpha_{k}\left(l^{1 / e}\right)^{e r}$, we have

$$
f_{z}\left(i\left(l^{1 / e}\right)\right)=\left\{\sum_{k=1}^{\infty} \alpha_{k} t^{\left(r_{k}-1\right)} / \Gamma\left(r_{k}\right)\right\}
$$

which is a element of $\mathcal{K}$ by Proposition 4.3. For other positive rational integer $e^{\prime}$ such that each $e^{\prime} r_{k}$ are integers, we have $f_{z}\left(i\left(l^{1 / e^{\prime}}\right)\right)=f_{z}\left(i\left(l^{1 / e}\right)\right)$, which shows that (4.8) is independent to $e$. So, we define $i(f)$ for any $f \in \mathscr{F}$ by

$$
i(f)=f_{z}\left(i\left(l^{1 / e}\right)\right) \text {. }
$$

Then we easily have the following proposition.

Proposition 4.4. For $f, g \in \mathscr{F}$ and $\alpha \in C$, we have

(1) $i(f)=i(g)$ if and only if $f=g$,

(2) $i(\alpha f)=\alpha i(f)$,

(3) $i(f+g)=i(f)+i(g)$,

(4) $i(f g)=i(f) i(g)$.

According to Proposition 4.4, the mapping $\imath$ is an injective ring homomorphism. By the Definition 3.3, we get the following identities for $f \in \mathscr{F}$.

$$
\begin{gathered}
i(l f)=\left\{\int_{0}^{t} i(f)(\tau) d \tau\right\} . \\
i\left(\partial_{t} f\right)=\left\{\frac{d}{d t} i(f)\right\} \quad \text { if } \nu(f)>1 .
\end{gathered}
$$

Proposition 4.5. For any $f \in \mathscr{F}$ and $\alpha \in C$, we have 


$$
i(f(l /(1-\alpha l)))=\left\{i(f) e^{\alpha t}\right\} .
$$

Proof. For $f=l^{r}(r>0)$, we get

$$
f(l /(1-\alpha l))=l^{r} /(1-\alpha l)^{r}=\sum_{k=0}^{\infty}\left(\begin{array}{c}
-r \\
k
\end{array}\right)(-\alpha)^{k} l^{k+r},
$$

which is an element of $\mathscr{I}$. Hence,

$$
\begin{aligned}
i(f(l /(1-\alpha l))) & =\left\{\sum_{k=0}^{\infty}\left(\begin{array}{c}
-r \\
k
\end{array}\right)(-\alpha)^{k} t^{k+r-1} / \Gamma(k+r)\right\} \\
& =\left\{\left(t^{r-1} / \Gamma(r)\right) \sum_{k=0}^{\infty}(\alpha t)^{k} / k !\right\}=\left\{\left(t^{r-1} / \Gamma(r)\right) e^{\alpha t}\right\} .
\end{aligned}
$$

So, for any $f=\sum_{k=1}^{\infty} \alpha_{k} l^{r} \in \mathscr{F}$, we get

$$
i(f(l /(1-\alpha l)))=\left\{\sum_{k=1}^{\infty} \alpha_{k}\left(t^{r_{k}-1} / \Gamma\left(r_{k}\right)\right) e^{\alpha t}\right\}=\left\{i(f) e^{\alpha t}\right\} .
$$

Applying Proposition 4.5, we show two examples.

EXAMPLE 1.

$$
i\left(1 /(s-\alpha)^{n}\right)=\left\{i\left(l^{n}\right) e^{\alpha t}\right\}=\left\{\left(t^{n-1} / \Gamma(n)\right) e^{\alpha t}\right\}
$$

EXAMPLE 2.

$$
\begin{aligned}
i(1 / \sqrt{s+\alpha}) & =i(\sqrt{l /(1+\alpha l)}) \\
& =\left\{(1 / \Gamma(1 / 2)) t^{(-1 / 2)} e^{-\alpha t}\right\} \\
& =\left\{(1 / \sqrt{\pi t}) e^{-\alpha t}\right\} .
\end{aligned}
$$

\section{Logarithmic roots.}

Let $k$ and $r$ be any positive rational numbers such that $k>r$ and $n$ the integer such that $k-n r>0$ and $k-(n+1) r \leqq 0$. We consider a following element $\omega$ of $C\{l\}$.

$$
\omega=\beta_{0} s^{k}+\beta_{1} s^{k-1}+\cdots+\beta_{n} s^{k-n r}+f,
$$

where $f \in \mathscr{I}, \beta_{i} \in C(i=0,1, \cdots, n), \beta_{0} \neq 0$ and $s=l^{-1}$.

For such an element $\omega$ of $\boldsymbol{C}\{l\}$, we have $\nu(\omega)=-k$. Hence, $l^{k+1} \omega \in \mathscr{F}$. Especially, any $f \in \mathscr{F}$ has no $s$-terms of positive power. In this case, we define $k=-\nu(f)<0$.

Let $x(\lambda, t)$ be a function of two variables $\lambda, t$ defined in interval $0<\lambda, t<\infty$. Suppose, 
(1) for every $t>0, x(\lambda, t)$ is differentiable with respect to $\lambda$,

(2) for every $\lambda>0, x(\lambda, t)$ and $\partial x / \partial \lambda(\lambda, t)$ are elements of $\mathcal{K}$.

For such a function $x(\lambda, t)$ and any $f \in \mathscr{F}$, we define

$$
i(f)\{x(\lambda, t)\}=\left\{\int_{0}^{t} i(f)(t-\tau) x(\lambda, \tau) d \tau\right\} \quad \text { for every } \lambda>0 .
$$

Definition 5.1. Let $\omega$ be an element of $C\{l\}$ given by (5.1) and $k=-\nu(\omega)$. Then $\omega$ is called a logarithmic root if the equation

$$
i\left(l^{|k|+1}\right)\left\{\frac{\partial x}{\partial \lambda}(\lambda, t)\right\}=i\left(l^{|k|+1} \omega\right)\{x(\lambda, t)\}
$$

has a solution $\{x(\lambda, t)\} \neq 0$ in the interval $0 \leqq \lambda, t<\infty$.

Remark that, if $k=-\nu(\omega)<0,(5.3)$ is equivalent to

$$
\left\{\frac{\partial x}{\partial \lambda}(\lambda, t)\right\}=i(\omega)\{x(\lambda, t)\},
$$

because of Proposition 4.4. For example, let $\omega=l$, then $x(\lambda, t)=\sum_{n=0}^{\infty}(t \lambda)^{n} /(n !)^{2}$ is the solution of $\{\partial x / \partial \lambda(\lambda, t)\}=i(l)\{x(\lambda, t)\}$ satisfying $x(0, t)=1$.

The next proposition is easily proved (cf. [2], p. 191).

Proposition 5.2. If $\omega$ is logarithmic, then for given $c \in \mathcal{F}(c \neq 0)$, there exists the unique solution satisfying the equation (5.3) and the condition $\{x(0, t)\}=i(c)$.

LEMMA 5.3. If $\omega_{1}, \omega_{2} \in \boldsymbol{C}\{l\}$ are logarithmic, then $\omega_{1}+\omega_{2}$ is also logarithmic and we have

$$
\begin{aligned}
& \left\{x_{\left(\omega_{1}+\omega_{2}\right)}(\lambda, t)\right\}=\left\{x_{\omega_{1}}(\lambda, t)\right\}\left\{x_{\omega_{2}}(\lambda, t)\right\} \\
& \text { and } \quad\left\{x_{\left(\omega_{1}+\omega_{2}\right)}(0, t)\right\}=\left\{x_{\omega_{1}}(0, t)\right\}\left\{x_{\omega_{2}}(0, t)\right\},
\end{aligned}
$$

where $x_{\omega_{1}}, x_{\omega_{2}}$ and $x_{\left(\omega_{1}+\omega_{2}\right)}$ are the solution of (5.3) corresponding to logarithmic roots $\omega_{1}, \omega_{2}$ and $\omega_{1}+\omega_{2}$, respectively.

Proof. Let us consider the equation

$$
i\left(l^{k^{\prime}+1}\right)\left\{\frac{\partial x}{\partial \lambda}(\lambda, t)\right\}=i\left(l^{k^{\prime}+1}\left(\omega_{1}+\omega_{2}\right)\right)\{x(\lambda, t)\}
$$

where $k^{\prime}=\left|\nu\left(\omega_{1}\right)\right|+\left|\nu\left(\omega_{2}\right)\right|$. Then $\left\{x_{\omega_{1}}(\lambda, t)\right\}\left\{x_{\omega_{2}}(\lambda, t)\right\}$ is the solution of (5.5) satisfying $\{x(0, t)\}=\left\{x_{\omega_{1}}(0, t)\right\}\left\{x_{\omega_{2}}(0, t)\right\}$. So, by Proposition 5.2, we have Lemma 5.3 .

From Proposition 5.2 and Lemma 5.3, we denote the solution of (5.3) satisfying the condition $\{x(0, t)\}=i(c)$ for $c \in \mathcal{F}$ as $\{X(\lambda, t ; \omega, c)\}$. Hence, (5.4) is rewritten as the following form, 


$$
\left\{X\left(\lambda, t ; \omega_{1}+\omega_{2}, c\right)\right\}=\left\{X\left(\lambda, t ; \omega_{1}, c_{1}\right)\right\}\left\{X\left(\lambda, t ; \omega_{2}, c_{2}\right)\right\}
$$

where $c, c_{1}$ and $c_{2} \in \mathscr{F}$ such that $c=c_{1} c_{2}$.

LEMMA 5.4. For $\omega \in \boldsymbol{C}\{l\}$ given by (5.1),

(1) if $k<0$, then $\omega$ is longarithmic,

(2) if $k>0$, then $\omega$ is logarithmic of and only if $\beta_{0} s^{k}$ is so.

Proof. (1) is obvious because of $\omega \in \mathcal{I}$, and (2) follows from Lemma 5.3.

The following lemma and examples are quoted from Mikusiński's results. So, we omit to give a full account.

LEMMA 5.5. If $k<1$, or $k=1$ and $\beta_{0}$ is real, then $\beta_{0} s^{k}$ is logarithmec (cf. [2], p. 399 p. 416)

EXAMPLE 1.

$$
\left\{X\left(\lambda, t ;-\sqrt{ } s, l^{1 / 2}\right)\right\}=\left\{(1 / \sqrt{ } \pi t) \exp \left(-\lambda^{2} /(4 t)\right)\right\} .
$$

EXAMPLE 2.

$$
\{X(\lambda, t ;-s, f)\}=h^{\lambda} \cdot i(f) \quad \text { for any } f \in \mathscr{I},
$$

where $h^{\lambda}$ is called translation operator which is given by

$$
h^{\lambda}\{i(f)(t)\}= \begin{cases}0 & \text { for } 0 \leqq t<\lambda \\ i(f)(t-\lambda) & \text { for } 0 \leqq \lambda<t .\end{cases}
$$

\section{Application to the partial differential equations.}

Let $\hat{k}$ be $\boldsymbol{C}\{l\}$ in section 2 and $\boldsymbol{C}\{l\}_{0}$ the set of all logarithmic roots. Because differential operator $S$ is transcendental over $C\{l\}$, we can consider the rational function field $\boldsymbol{C}\{l\}(S)$, which has the unique partially fractional decomposition. From Lemma 2.5, we get $c /(S-\omega)^{n}=\left\{c\left(\lambda^{n-1} /(n-1) !\right) e^{\omega \lambda}\right\} \in \mathcal{A}$, where $\omega \in \boldsymbol{C}\{l\}$ and $c \in \mathscr{I}$. Let $\mathcal{A}_{0}$ be the subset of $\mathcal{A}$ given by the following.

$$
\mathcal{A}_{0}=\left\{\sum_{\omega} \sum_{n=1}^{n_{\omega}} \frac{c_{\omega, n}}{(S-\omega)^{n}} \quad \text { (finite sum), } \omega \in C\{l\}_{0}, c_{\omega, n} \in \mathscr{F}\right\} .
$$

Then $\mathcal{A}_{0}$ is a $\mathscr{I}$-module. For any $\omega \in \boldsymbol{C}\{l\}_{0}$ and $c \in \mathscr{F}$, we can get the unique function $\{X(\lambda, t ; \omega, c)\}$ of two variables, which is the solution of (5.3) satisfying $\{x(0, t)\}=i(c)$. For each $\left\{c e^{\omega \lambda}\right\}$, we define a function $j\left(c e^{\omega \lambda}\right)$ of two variables $\lambda, t$ as the following.

$$
j\left(c e^{\omega \lambda}\right)=\{X(\lambda, t ; \omega, c)\} .
$$

Now, the mapping $\partial_{\lambda}$ and $\partial_{t}$ operate in $\mathcal{A}$ as the following (cf. Def. 2.3 and 
Def. 3.3). For any $P=\left\{\sum_{\nu=0}^{\infty} p_{\nu} \lambda^{\nu}\right\} \in \mathcal{A}\left(p_{\nu} \in C\{l\}\right)$,

$$
\begin{gathered}
\partial_{\lambda}(P)=\left\{p_{1}+2 p_{2} \lambda+3 p_{3} \lambda^{2}+\cdots\right\}, \\
\partial_{t}(P)=\left\{\partial_{t}\left(p_{0}\right)+\partial_{t}\left(p_{1}\right) \lambda+\partial_{t}\left(p_{2}\right) \lambda^{2}+\cdots\right\} .
\end{gathered}
$$

By the definition of $j\left(c e^{\omega \lambda}\right)$, we have

$$
\begin{aligned}
& i\left(l^{k+1}\right) \frac{\partial}{\partial \lambda} j\left(c e^{\omega \lambda}\right)=i\left(l^{k+1} \omega\right) j\left(c e^{\omega \lambda}\right) \\
& \text { and } j\left(c e^{\omega \lambda}\right)(0, t)=i(c),
\end{aligned}
$$

where $k=|\nu(\omega)|$. By differentiating partially (6.1) with respect to $\lambda$, we get

$$
i\left(l^{k+1}\right) \frac{\partial}{\partial \lambda}\left\{\frac{\partial}{\partial \lambda} j\left(c e^{\omega \lambda}\right)\right\}=i\left(l^{k+1} \omega\right) \frac{\partial}{\partial \lambda} j\left(c e^{\omega \lambda}\right)
$$

and

$$
\left(i\left(l^{k+1}\right) \frac{\partial}{\partial \lambda} j\left(c e^{\omega \lambda}\right)\right)(0, t)=i\left(l^{k+1} \omega c\right)
$$

Because of the uniqueness of solution, (6.2) and (6.3) show that $\frac{\partial}{\partial \lambda} j\left(c \omega e^{\omega \lambda}\right)$ $=j\left(c \omega e^{\omega \lambda}\right)$. On the other hand, we have

Hence, we have

$$
j\left(\partial_{\lambda}\left(c e^{\omega \lambda}\right)\right)=j\left(c \omega e^{\omega \lambda}\right) .
$$

$$
\frac{\partial}{\partial \lambda} j\left(c e^{\omega \lambda}\right)=j\left(\partial_{\lambda}\left(c e^{\omega \lambda}\right)\right)
$$

Here, we assume $\nu(c)>1$ because of $\nu(l c)>1$. By differentiating partially (6.1) with respect to $t$ and the property of the convolution, the left hand side of (6.1) becomes

or

$$
\begin{aligned}
\frac{\partial}{\partial t}\left(i\left(l^{k+1}\right) \frac{\partial}{\partial \lambda} j\left(c e^{\omega \lambda}\right)\right) & =\left\{\frac{\partial}{\partial t} i\left(l^{k+1}\right)\right\}\left\{\frac{\partial}{\partial \lambda} j\left(c e^{\omega \lambda}\right)\right\}=i\left(l^{k}\right) \frac{\partial}{\partial \lambda} j\left(c e^{\omega \lambda}\right) \\
& =i\left(l^{k+1}\right) \frac{\partial}{\partial \lambda} \cdot \frac{\partial}{\partial t}\left(j\left(c e^{\omega \lambda}\right)\right)
\end{aligned}
$$

On the other hand, the right hand side becomes

or

$$
\begin{aligned}
\frac{\partial}{\partial t}\left(i\left(l^{k+1} \omega\right) j\left(c e^{\omega \lambda}\right)\right) & =\left(\frac{\partial}{\partial t} i\left(l^{k+1} \omega\right)\right) j\left(c e^{\omega \lambda}\right)=i\left(l^{k} \omega\right) j\left(c e^{\omega \lambda}\right) \\
& =i\left(l^{k+1} \omega\right) \frac{\partial}{\partial t} j\left(c e^{\omega \lambda}\right) .
\end{aligned}
$$

Combining (1) and (3), or (2) and (4), we get that $j\left(c s e^{\omega \lambda}\right)$ or $\frac{\partial}{\partial t} j\left(c e^{\omega \lambda}\right)$ is 
the solution of the equation (5.3) satisfying $\{x(0, t)\}=i(s c)$ or $\{\partial / \partial t i(c)\}$, respectively. By (4.11), we have $i(s c)=\{\partial / \partial t i(c)\}$, which shows that

$$
\frac{\partial}{\partial t} j\left(c e^{\omega \lambda}\right)=j\left(c s e^{\omega \lambda}\right) \text {. }
$$

On the other hand, $j\left(\partial_{t}\left(c e^{\omega \lambda}\right)\right)=j\left(s c e^{\omega \lambda}\right)$. Hence, we have

$$
\frac{\partial}{\partial t} j\left(c e^{\omega \lambda}\right)=j\left(\partial_{t}\left(c e^{\omega \lambda}\right)\right) \text {. }
$$

For $\left\{\left(c \lambda^{n-1} /(n-1) !\right) e^{\omega \lambda}\right\} \in \mathcal{A}_{0}$, we define $j\left(\left(c \lambda^{n-1} /(n-1) !\right) e^{\omega \lambda}\right)$ as the following

$$
j\left(\frac{c \lambda^{n-1}}{(n-1) !} e^{\omega \lambda}\right)=\left\{\frac{\lambda^{n-1}}{(n-1) !} j\left(c e^{\omega \lambda}\right)\right\}
$$

and for any $P=\left\{\sum_{\omega} \sum_{n=1}^{n_{\omega}} c_{\omega, n} /(S-\omega)^{n}\right\} \in \mathcal{A}_{0}$, we define $j(P)$ as the following

$$
j(P)=\sum_{\omega} \sum_{n=1}^{n_{(\prime \prime}} j\left(\frac{c_{\omega, n} \lambda^{n-1}}{(n-1) !} e^{\omega \lambda}\right) .
$$

Then we put $\mathscr{B}_{0}=\left\{j(P)\right.$; any $\left.P \in \mathcal{A}_{0}\right\}$, which is some set of functions of two variables $\lambda, t$. Then, by virtue of (6.4) and (6.6), we get

$$
\begin{aligned}
& j\left(\partial_{\lambda}\left(\frac{c \lambda^{n-1}}{(n-1) !} e^{\omega \lambda}\right)\right)=\frac{\partial}{\partial \lambda}\left(j\left(\frac{c \lambda^{n-1}}{(n-1) !} e^{\omega \lambda}\right)\right), \\
& j\left(\partial_{t}\left(\frac{c \lambda^{n-1}}{(n-1) !} e^{\omega \lambda}\right)\right)=\frac{\partial}{\partial t}\left(j\left(\frac{c \lambda^{n-1}}{(n-1) !} e^{\omega \lambda}\right)\right) .
\end{aligned}
$$

Hence, the following diagrams are commutative.
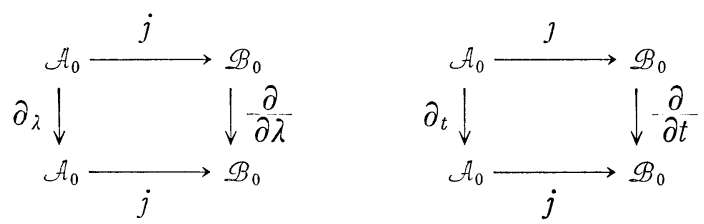

Now, we consider the linear partial differential equation of two variables $\lambda, t$ with constant coefficients. Any such equation can be written in the form,

$$
\sum_{\mu=0}^{m} \sum_{\nu=0}^{n} \alpha_{\mu \nu} \frac{\partial^{\mu+\nu} x}{\partial \lambda^{\mu} \partial t^{\nu}}(\lambda, t)=\varphi(\lambda, t) \quad\left(\alpha_{\mu \nu} \in \boldsymbol{C}\right)
$$

We assume that there exists $Y \in \mathcal{A}_{0}$ such that $j(Y)=\{\varphi(\lambda, t)\}$. Then, we consider the following operational equation in $A$.

$$
\sum_{\mu=0}^{m} \sum_{\nu=0}^{n} \alpha_{\mu \nu} \partial_{\lambda}^{\mu} \partial_{t}^{\nu} X=Y
$$


From Proposition (3.4), we have

$$
\partial_{t}^{\nu}\left(\partial_{\lambda}^{\mu} X\right)=s^{\nu} \partial_{\lambda}^{\mu} X-\sum_{k=0}^{\nu-1} s^{\nu-k-1} \partial_{t}^{k} \partial_{\lambda}^{\mu} X(\lambda, 0),
$$

where we assume that $\partial_{t}^{k} \partial_{\lambda}^{\mu} X(\lambda, 0) \in \mathcal{A}_{0}$ are determined by the equation

$$
j\left(\partial_{t}^{k} \partial_{\lambda}^{\mu} X(\lambda, 0)\right)=\left\{\frac{\partial^{k+\mu} x}{\partial t^{k} \partial \lambda^{\mu}}(\lambda, 0)\right\} \quad(k=0,1, \cdots, \nu-1),
$$

which are called initial conditions of (6.10).

Hence, (6.11) becomes

$$
a_{m} \partial_{\lambda}^{m} X+a_{m-1} \partial_{\lambda}^{m-1} X+\cdots+a_{0} X=F,
$$

where $a_{\mu}=\alpha_{\mu n} s^{n}+\cdots+\alpha_{\mu 0}(\mu=0,1, \cdots, m)$ and

$$
F=Y+\sum_{\mu=0}^{m} \sum_{\nu=1}^{n} \sum_{k=0}^{\nu-1} \alpha_{\mu \nu} s^{\nu-k-1} \partial_{\lambda}^{\mu} \partial_{t}^{k} X(\lambda, 0) \in \mathcal{A} .
$$

Applying the operational calculus (cf. section 2) with respect to $\lambda$ to (6.13), we have the following.

$$
\left(a_{m} S^{m}+a_{m-1} S^{m-1}+\cdots+a_{0}\right) X=b_{m-1} S^{m-1}+\cdots+b_{0}+F,
$$

where $b_{m-1}, \cdots, b_{0}$ are determined by the values of

$$
x(0, t), \frac{\partial x}{\partial \lambda}(0, t), \cdots, \frac{\partial^{m-1} x}{\partial \lambda^{m-1}}(0, t),
$$

which are called boundary conditions.

Since $\hat{k}=\boldsymbol{C}\{l\}$ is algebrically closed, we can decompose

$$
a_{m} S^{m}+\cdots+a_{0}=a_{m} \prod_{\imath=1}^{m}\left(S-\omega_{i}\right) \quad\left(\omega_{i} \in \boldsymbol{C}\{l\}\right)
$$

Hence, by simple-fraction-decomposition method, we can get the solution of (6.11) in $\mathcal{A}$ (cf. section 2).

If $\omega$ is not logarithmic, choosing suitable boundary conditions, we can neglect these parts. Thus we can get the solution $X_{0}$ in $\mathcal{A}_{0}$. Because of the commutative diagrams (6.9), we get the solution $j\left(X_{0}\right)$ of $(6.10)$.

\section{Examples.}

1. Solve the following differential equation (7.1) with the conditions (7.2).

$$
\begin{aligned}
& \frac{\partial^{2} x}{\partial \lambda^{2}}-\frac{\partial^{2} x}{\partial t^{2}}=0 \quad(0 \leqq \lambda, t<\infty) . \\
& x(\lambda, 0)=\lambda^{2}, \quad \frac{\partial x}{\partial t}(\lambda, 0)=3 \lambda^{2}-2 \lambda,
\end{aligned}
$$




$$
x(0, t)=t^{3}, \quad \frac{\partial x}{\partial \lambda}(0, t)=0 .
$$

By virtue of (6.9), (7.1) is equivalent to

$$
\partial_{\lambda}^{2} X-\partial_{t}^{2} X=0 \text {. }
$$

Here, from Proposition (3.4) and condition (7.2), we have

$$
\partial_{t}^{2} X=s^{2} X-s \cdot x(\lambda, 0)-\frac{\partial x}{\partial t}(\lambda, 0)=s^{2} X-s \lambda^{2}-3 \lambda^{2}+2 \lambda .
$$

Hence, (7.3) becomes

$$
\partial_{\lambda}^{2} X-s^{2} X=-s \lambda^{2}-3 \lambda^{2}+2 \lambda .
$$

Next, applying the operational calculus with respect to $\lambda,(7.5)$ is written as

$$
S^{2} X-s^{2} X=6 l^{4} S-\frac{(2 s+6)}{S^{3}}+\frac{2}{S^{2}} .
$$

From which, we have

$$
\begin{aligned}
X & =\frac{6 l^{4} S}{S^{2}-s^{2}}-\frac{(2 s+6)}{S^{3}\left(S^{2}-s^{2}\right)}+\frac{2}{S^{2}\left(S^{2}-s^{2}\right)} \\
& =\frac{-2 l^{3}}{S+s}+\frac{\left(2 l^{3}+6 l^{4}\right)}{S}-\frac{2 l^{2}}{S^{2}}+\frac{\left(2 l+6 l^{2}\right)}{S^{3}} .
\end{aligned}
$$

Because $1 /(S+s)=e^{-s \lambda}$ is translation operator (cf. section 5 , example 2), we obtain

$$
x(\lambda, t)= \begin{cases}3 \lambda^{2} t+\lambda^{2}-2 \lambda t+t^{2}+t^{3} & 0 \leqq t<\lambda \\ 3 \lambda^{2} t+t^{3} & 0 \leqq \lambda<t .\end{cases}
$$

This is the only solution which satisfies (7.1) and (7.2) in the interval $0 \leqq \lambda, t<\infty$.

2. Solve the following differential equation (7.8) with the conditions (7.9).

$$
\begin{gathered}
\frac{\partial^{2} x}{\partial \lambda^{2}}+\frac{\partial^{2} x}{\partial t^{2}}=1 . \\
x(\lambda, 0)=0, \quad x_{t}(\lambda, 0)=\lambda^{2} e^{-\lambda} .
\end{gathered}
$$

By the same way as example 1, we have the following operational equation.

$$
S^{2} X+s^{2} X=c_{1} S+c_{2}+l L+\frac{2}{(S+1)^{3}},
$$

where $c_{1}$ and $c_{2}$ are undetermined constants. From which, we get 


$$
\begin{aligned}
X= & \frac{c_{1}^{\prime} S+c_{2}^{\prime}}{\left(S^{2}+s^{2}\right)}+\frac{\left(-2 s^{2}+6\right)}{(s+1)^{3}} \cdot \frac{1}{(S+1)}+\frac{4}{\left(s^{2}+1\right)^{2}} \cdot \frac{1}{(S+1)^{2}} \\
& +\frac{2}{\left(s^{2}+1\right)} \cdot \frac{1}{(S+1)^{3}}+\frac{l^{3}}{S} .
\end{aligned}
$$

Here, $\pm i$ s are not logarithmic. Choosing suitable boundary conditions, we can get $c_{1}^{\prime}=c_{2}^{\prime}=0$. Hence, (7.11) becomes

$$
\begin{aligned}
X= & {\left[\frac{-2}{\left(s^{2}+1\right)^{2}}+\frac{8}{\left(s^{2}+1\right)^{3}}\right] \cdot \frac{1}{(S+1)}+\frac{4}{\left(s^{2}+1\right)^{2}} \cdot \frac{1}{(S+1)^{2}} } \\
& +\frac{2}{\left(s^{2}+1\right)} \cdot \frac{1}{(S+1)^{3}}+\frac{l^{3}}{S} .
\end{aligned}
$$

From which, we have

$$
\begin{aligned}
x(\lambda, t)= & {\left[-(\sin (t)-t \cos (t))+2\left(\left(\frac{3}{2}-\frac{t^{2}}{2}\right) \sin (t)-\frac{3}{2} t \cdot \cos (t)\right)\right] e^{-\lambda} } \\
& +2(\sin (t)-t \cos (t)) \lambda e^{-\lambda}+2 \sin (t) \frac{\lambda^{2}}{2} e^{-\lambda}+\frac{t^{2}}{2} \\
= & {\left[\left(2-t^{2}\right) \sin (t)-2 \cos (t)+2 \lambda(\sin (t)-t \cos (t))+\lambda^{2} \sin (t)\right] e^{-\lambda}+\frac{t^{2}}{2} . }
\end{aligned}
$$

which is the solution of (7.8) satisfying (7.9).

3. Solve the following differential equation (7.12) with the conditions (7.13).

$$
\begin{gathered}
\frac{\partial^{4} x}{\partial \lambda^{2} \partial t^{2}}-2 \frac{\partial^{3} x}{\partial \lambda^{2} \partial t}+\frac{\partial^{2} x}{\partial \lambda^{2}}=x-4 e^{\lambda} \quad(0 \leqq \lambda, t<\infty) \\
x_{\lambda \lambda}(\lambda, 0)=e^{\lambda}, \quad x_{\lambda \lambda t}(\lambda, 0)-2 x_{\lambda \lambda}(\lambda, 0)=\lambda, \\
x(0, t)=1+2 t, \quad x_{\lambda}(0, t)=2 t .
\end{gathered}
$$

By the same way as example 1 and 2, we have the following operational equation.

$$
(s-1)^{2} S^{2} X-X=(s-1)^{2}\left(\left(l+2 l^{2}\right) S+2 l^{2}\right)+\frac{(s-4 l)}{S-1}+\frac{1}{S^{2}} .
$$

From which, we have

$$
\begin{aligned}
X & =\frac{(s-1)^{2}\left(\left(l+2 l^{2}\right) S+2 l^{2}\right)}{(s-1)^{2} S^{2}-1}+\frac{(s-4 l)}{\left((s-1)^{2} S^{2}-1\right)(S-1)}+\frac{1}{S^{2}\left((s-1)^{2} S^{2}-1\right)} \\
& =\frac{l(s-1)^{2}}{2(S-1 /(s-1))}-\frac{l(s-1)^{2}}{2(S+1 /(s-1))}+\frac{\left(l+2 l^{2}\right)}{(S-1)}-\frac{1}{S^{2}}
\end{aligned}
$$


where $\pm 1 /(s-1)= \pm\left\{e^{t}\right\}$ are logarithmic. By the some routine calculations, we have the solution of (7.12) satisfying (7.13).

$$
x(\lambda, t)=(1+2 t) e^{\lambda}-\lambda-\sum_{\nu=1}^{\infty} \frac{\lambda^{2 \nu+1}}{(2 \nu+1) !}\left[1+e^{t}\left(1-\frac{t}{1}+\cdots+\frac{t^{2 \nu-2}}{(2 \nu-2) !}\right)\right] .
$$

\section{REFERENCES}

[1] K. IwaSAwa, Daisū kansūron (in Japanese, Theory of algebraic functions), Iwanami (1952).

[2] J. Mikusiński, Operational calculus, Pergamon Press (1959).

Department of Mathematics

YAMAGATA UNIVERSITY

YAMAGATA, JAPAN 